\title{
The effect of laser energy on the preparation of iron oxide by a pulsed laser ablation in ethanol
}

\author{
P. Maneeratanasarn, T.V. Khai, B.G. Choi and K.B. Shim ${ }^{\dagger}$ \\ Division of Advanced Material Science and Engineering, Hanyang University, Seoul 133-791, Korea \\ (Received February 6, 2012) \\ (Revised April 24, 2012) \\ (Accepted April 27, 2012)
}

\begin{abstract}
Recently the preparation magnetic nanoparticles by a pulsed laser ablation in liquid has gained much attention because it is easy to control experimental parameters. Iron oxide magnetic nanoparticles have been prepared by a pulsed laser ablation of $\alpha-\mathrm{Fe}_{2} \mathrm{O}_{3}$ target in ethanol at different magnitude of laser energy of $1,20,40$ and $80 \mathrm{~mJ} / \mathrm{pulse}$. It revealed that particle size increases with increasing laser energy. It could be concluded that $40 \mathrm{~mJ} / \mathrm{pulse}$ is an optimum laser energy for the preparation of iron oxide nanoparticles with uniform size distribution. The nanoparticles are homogeneously dispersed in ethanol and their stability maintained for several months.
\end{abstract}

Key words Iron oxide, Maghemite, Pulse laser ablation

\section{Introduction}

During last decade, a liquid phase pulse laser ablation (LP-PLA) has been extensively employed for the fabrication of nanoparticles because this technique is simple, clean and low cost. In particular, this technique can provide a favorable processing condition for preparing metastable phases and even novel nanomaterials; hightemperature, high-pressure and high density [1]. Therefore, the LP-PLA can be adapted for various nanoparticles using different processing conditions such as targets, solvents and processing parameters (pulse duration, fluence and laser wavelength, etc.) Many reports were found on the formation of various type of nanoparticles by the LP-PLA technique on such as metals [2, 3], alloys [4-6], oxides [7-9], and other nanocrystals [10]. More recently, the LP-PLA preparation of magnetic nanoparticles such as CoPt [11], SmCo, NiFe [12], FeCo [13], Co [14], and iron oxide [15] has been paid much attention.

Iron oxide materials possess magnetic character which has great potential for various applications such as recording and information storage $[16,17]$, magenetic shield case [18], catalyst [19] biomedical [20-23] and photochemical and photoelectochemical [23]. Amendola et al. reported on the fabrication of iron based magnetic

\footnotetext{
Corresponding author

Tel: +82-2-2220-0501

Fax: +82-2-2291-7395

E-mail: kbshim@hanyang.ac.kr
}

nanoparticles by the PLA in various organic solvents. This work emphasized that a variety of magnetic nanostructures can be produced by varying type of solvents [15]. This work is aimed to understanding the effect of laser energy on the characteristics of iron oxide nanoparticles prepared by the LP-PLA.

\section{Experimental}

Commercial $\alpha-\mathrm{Fe}_{2} \mathrm{O}_{3}$ powders were uni-axially pressed for 10 minutes in disc-shaped mold. The pressed $\alpha$ $\mathrm{Fe}_{2} \mathrm{O}_{3}$ pellets were sintered at $1400^{\circ} \mathrm{C}$ for 20 hours under ambient atmosphere using a conventional electric furnace. Then the sintered $\alpha-\mathrm{Fe}_{2} \mathrm{O}_{3}$ targets were ablated by the laser beam with $2 \mathrm{~mm}$-diameter focal spot size (Nd: YAG nanosecond laser: Quanta Ray-LAB190, USA) for one hour in $4 \mathrm{ml}$ of ethanol. The repetition rate, pulse duration and laser wavelength are $30 \mathrm{~Hz}, 10 \mathrm{~ns}$ and $355 \mathrm{~nm}$, respectively. The laser energy were varied 1, 20, 40, $80 \mathrm{~mJ} / \mathrm{pulse}$.

Optical property of the obtained nanocolloidal solutions was evaluated using a UV-VIS-NIR spectrophotometer (UV-3600, SHIMADZU, Japan). Structural characteristics of the obtained nanoparticles were investigated using a transmission electron microscope (TEM, JEM 2000 EXII, JEOL, Japan) and their crystallinity and phase analysis were examined using a X-ray diffractometer (XRD, D/ MAX2C, Rigaku, Japan). The average size and size distribution of as-processed nanoparticle were analyzed by 
using image analysis program, Image $\mathrm{J}$, and using standard statistic equations for calculating standard deviation and mean.

Magnetic characterization of these nanoparticles were carried out using vibrating sample magnetometer (VSM, Lakeshore 340, USA) at room temperature $(300 \mathrm{~K})$ by saturating the sample in a field of 5000 Oe.

\section{Results and Discussion}

Figure 1 shows TEM images of nanoparticles obtained in ethanol, depending on the laser energy of 1, 20, 40 and $80 \mathrm{~mJ} /$ pulse, respectively. Inserted SAED patterns confirm that all nanoparticles are crystalline. As-synthe- sized nanoparticles at very low laser energy of $1 \mathrm{~mJ} /$ pulse are seen as irregularly-shaped and about $8 \mathrm{~nm}$ in an average size. Some particles are characterized by a core-shell structure and also agglomerated as shown in Fig. 1(a)left-right. As laser energy increases to $20 \mathrm{~mJ} /$ pulse, the spherical and agglomerated nanoparticles were observed (Fig. 1(b)). The size distribution exhibits bimodal with a maximum values near $11 \mathrm{~nm}$ and $20 \mathrm{~nm}$. At laser energy of $40 \mathrm{~mJ} /$ pulse, individual spherical nanopaticles with an average size of $11 \mathrm{~nm}$ were obtained and are rarely agglomerated (Fig. 1(c)). The laser energy of $80 \mathrm{~mJ} /$ pulse resulted in forming chain-like nanoparticles having average size of $15 \mathrm{~nm}$ (Fig, 1(d)). These illustrates that the average particle size increases with increasing laser energy, which is in good agreement
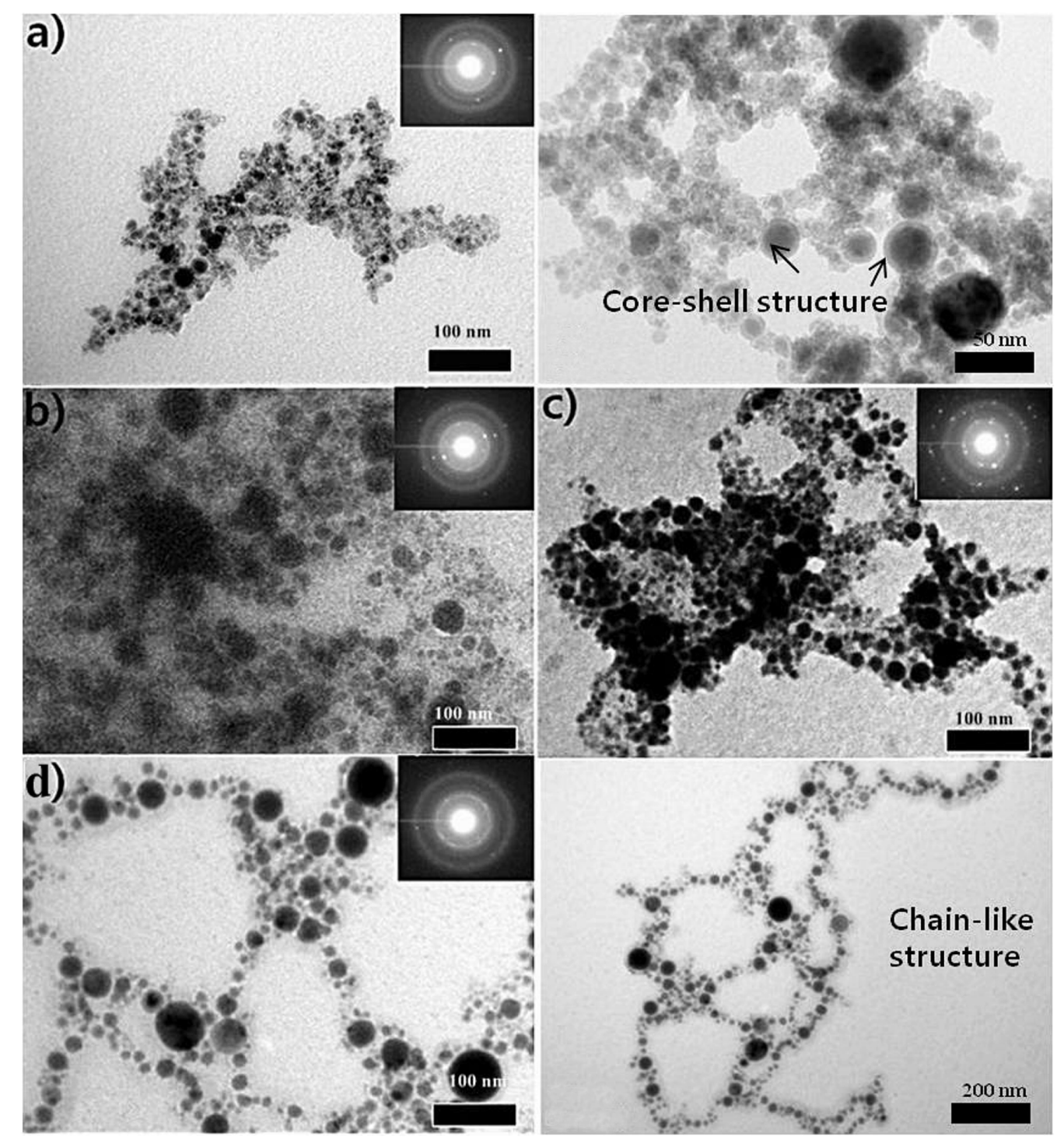

Fig. 1. TEM-SAED images of nanoparticles obtained by the ablation of $\alpha-\mathrm{Fe}_{2} \mathrm{O}_{3}$ targets in ethanol at a) $\left.1 \mathrm{~mJ} / \mathrm{pulse} \mathrm{b}\right) 20 \mathrm{~mJ} / \mathrm{pulse}$ c) $40 \mathrm{~mJ} /$ pulse d) $80 \mathrm{~mJ} /$ pulse, respectively. 

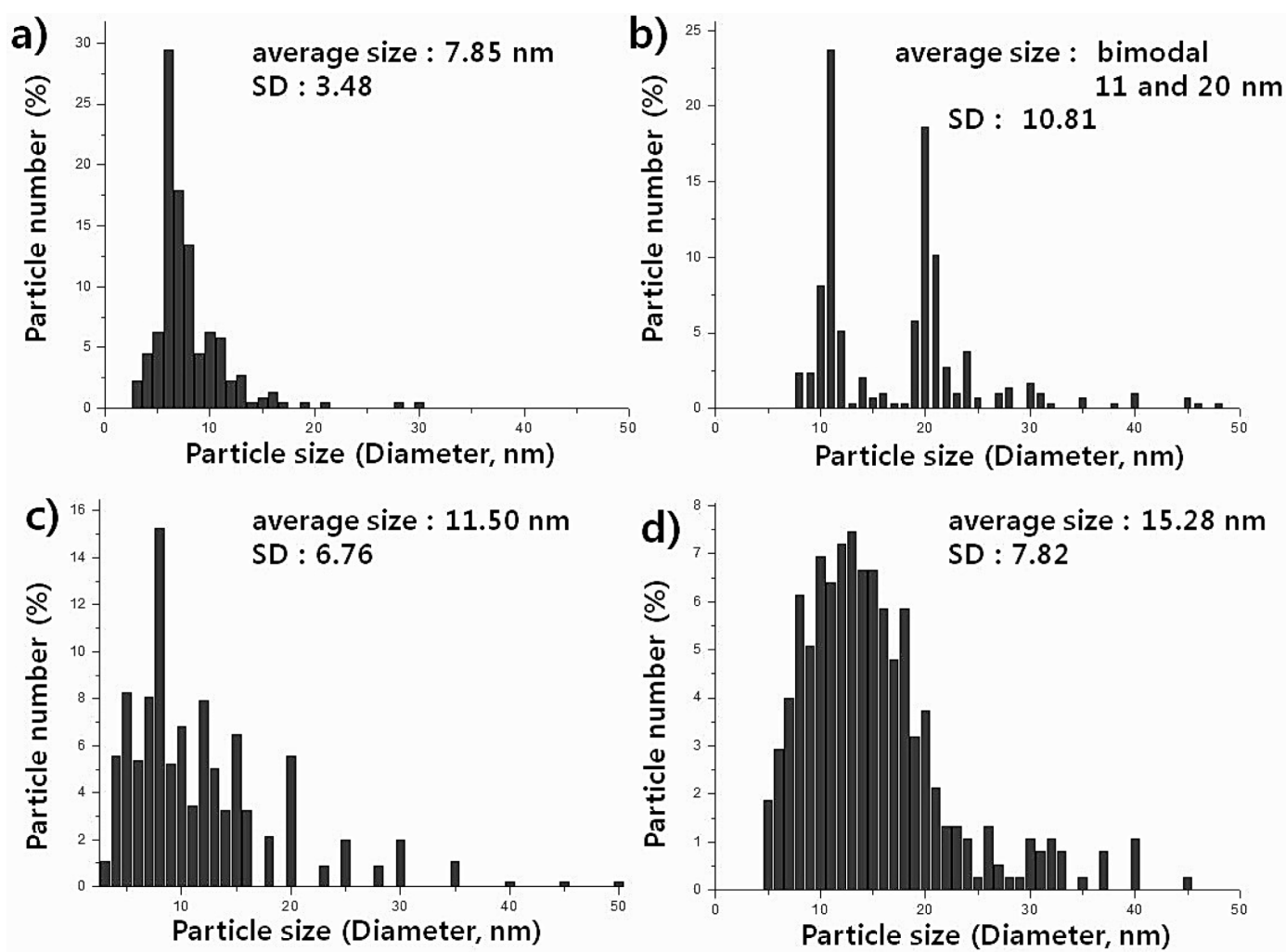

Fig. 2. Average size and particle size distribution with standard deviation value of nanoparticles obtained by the ablation of $\alpha-\mathrm{Fe}_{2} \mathrm{O}_{3}$ targets in ethanol at a) $1 \mathrm{~mJ} /$ pulse b) $20 \mathrm{~mJ} /$ pulse c) $40 \mathrm{~mJ} /$ pulse d) $80 \mathrm{~mJ} /$ pulse, respectively.

with previous reports $[24,25]$.

Figure 2 shows particle size distribution and average size of nanoparticles obtained in ethanol, depending on the laser energy of 1, 20, 40 and $80 \mathrm{~mJ} /$ pulse, respectively.

Although standard deviation (SD) value and average size of the nanoparticles obtained at laser energy of $1 \mathrm{~mJ} / \mathrm{pulse}$ is less than that obtained at laser energy of $40 \mathrm{~mJ} /$ pulse, indicating small particle size with good size distribution, but it causes high degree of agglomeration. Thus the laser energy of $40 \mathrm{~mJ} / \mathrm{pulse}$ is optimum condition in order to produce the nanoparticles with less agglomeration and uniform size distribution.

According to the experimental results that higher laser energy produces higher amount of species from the ablated target in plasma plume [26-29], the formation of larger nuclei may occur in a condensation step. Also excessive iron and oxygen atoms in plasma plume involve in severe growth of the nanoparticles [24]. In case of the formation of chain-like nanoparticles obtained at $80 \mathrm{~mJ} / \mathrm{pulse}$, it can be noticed that large particles (more than $10 \mathrm{~nm}$ in diameter) are stuck in ensembles of 300$400 \mathrm{~nm}$ length and surrounded by small particles (less than 10 in diameter). This is because small particles, which are very reactive resulting from high surface energy, react with adjacent small particles leading coag- ulation. This coagulation surrounding large particles drives the formation of chain-like nanoparticles. It could be simply explained that the coagulation of small particles is like glue which assists large particles to stick in ensembles as a chain-like shape.

Figure 3 shows the XRD results that all nanoparticles obtained depending on laser energy. Mainly tetragonal maghemite $\left(\gamma-\mathrm{Fe}_{2} \mathrm{O}_{3}\right)$ and carbon $(\mathrm{C})$ are detected. Their crystallinity increases with increasing laser energy. Dur-

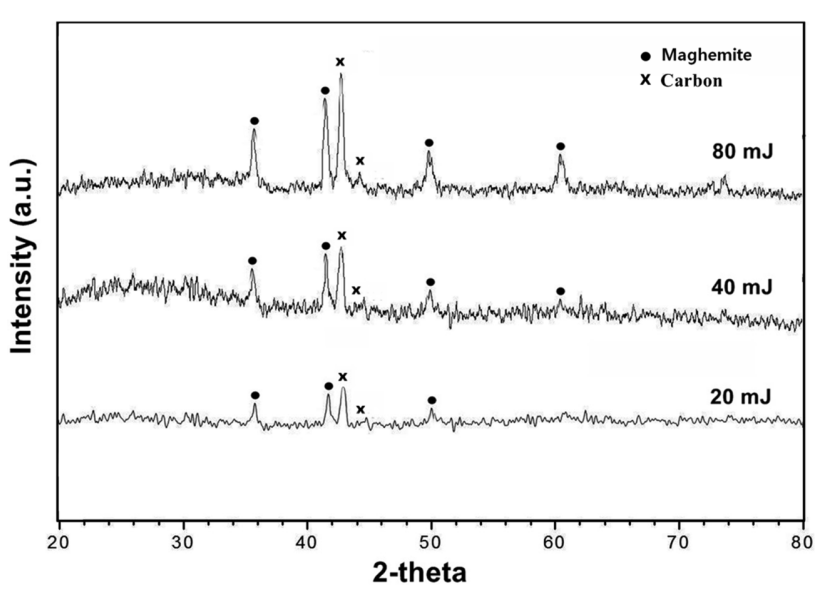

Fig. 3. X-ray diffraction patterns of nanoparticles obtained in ethanol, depending on laser energy. 
ing a laser ablation process of $\alpha-\mathrm{Fe}_{2} \mathrm{O}_{3}$ targets, $\mathrm{Fe}$ plasma reacted with oxygen species, which were originated from the ablated target and ethanol, to form $\gamma-\mathrm{Fe}_{2} \mathrm{O}_{3}$ at extremly high temperature and high prssure. In general, $\gamma-\mathrm{Fe}_{2} \mathrm{O}_{3}$ are formed above $1200^{\circ} \mathrm{C}$ [30]. Carbon phase is also possibly released from ethanol during the laser ablation process.

All nanocolloidal solutions obtained are yellowish with different shade. Figure 4 shows their representative UV-Vis spectra depending on laser energy. Nanocolloidal solutions obtained at 20, 40 and $80 \mathrm{~mJ} /$ pulse exhibit a broad absorption band near 300-400 nm [31]. The intensities increase as laser energy increases, indicating that the concentration of as-processed nanoparticles increases with increasing laser energy [24]. In case of $1 \mathrm{~mJ} /$

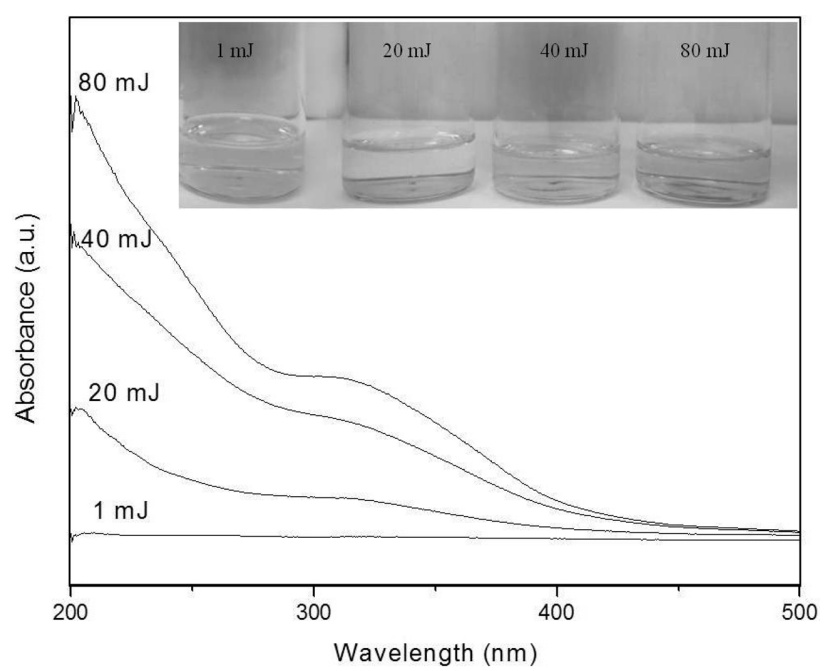

Fig. 4. UV-Vis absorption spectra of nanocolloidal solutions produced by laser ablation of $\alpha-\mathrm{Fe}_{2} \mathrm{O}_{3}$ target in ethanol, depending on laser energy.

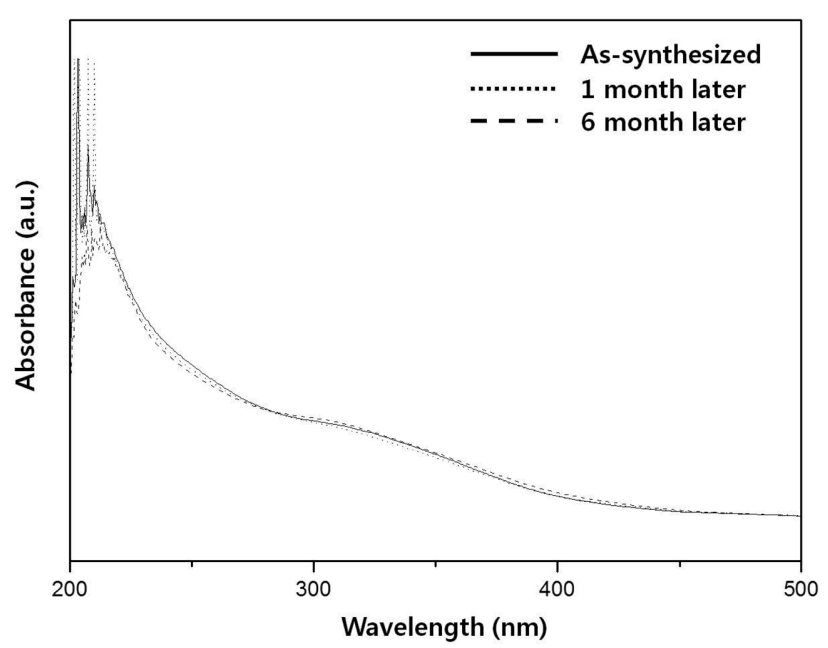

Fig. 5. UV-Vis spectra of nanocolloidal solution obtained at laser energy of $40 \mathrm{~mJ} /$ pulse for the evaluation of the stability.

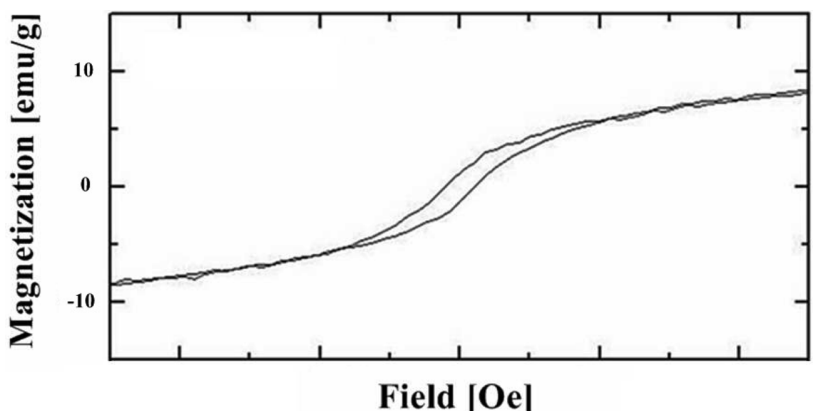

Fig. 6. Magnetic hysteresis curves measured at room temperature $(300 \mathrm{~K})$ of nanoparticles extracted from nanocolloidal solutions obtained at laser energy of $40 \mathrm{~mJ} /$ pulse for the evaluation of magnetic character.

pulse laser energy, there is no any broad absorption band, which is attributed by that low laser energy yield too low concentration of the nanoparticles to be examined.

Figure 5 shows UV-Vis spectra of the obtained nanocolloidal solution for the evaluation of their stability. It is seen that there is no change even after 6 months, indicating that these colloidal solutions are stable for several months. This stability may be attributed by the presence of group - $\mathrm{OH}$, forming stable interaction with the iron oxide nanoparticles [32].

Figure 6 illustrates the magnetization curve measured at $300 \mathrm{~K}$ for nanoparticles obtained in ethanol at the laser energy $40 \mathrm{~mJ} /$ pulse for the assessment of their magnetic character. The coercivity, remanent magnetization and magnetization of these nanoparticles are $145.56 \mathrm{Oe}, 1.45$ emu/g and $5.59 \mathrm{emu} / \mathrm{g}$, respectively. This confirms that the obtained nanoparticles exhibit magnetic characteristics.

\section{Conclusions}

Iron oxide nanoparticles with good size distribution were obtained by a pulsed laser ablation of metal target in ethanol and $40 \mathrm{~mJ} /$ pulse was found as optimum laser energy. It is noted that laser energy plays an important role in particle size and size distribution. Particle size increases with increase of the laser energy. The formation of carbon is inevitable because the solvents themselves are source of free carbon. In our view, use of other solvents such water is probably a solution for this insignificant drawback due to no carbon source. However, in order to extend this knowledge for better comprehension, further experimental study is necessarily required. The nanoparticles obtained possess magnetic characteristics and therefore they are expected to be used in magnetic applications such as recording and 
information storage, biomedical and photoelectochemical applications.

\section{Acknowledgements}

We wish to gratefully acknowledge with sincere thanks BK 21 and National Research Foundation of Korea for the financial support.

\section{References}

[1] G.W. Yang, "Laser ablation in liquids: Applications in the synthesis of nanocrystals", Progress in materials Science 52 (2007) 648.

[2 ] T. Tsuji, et al., "Preparation of nano-size particles of silver with femtosecond laser ablation in water", Applied Surface Science 206 (2003) 314.

[ 3 ] G. Compagini, et al., "Production of gold nanoparticles by laser ablation in liquid alkanes", J. Appl. Phys. 94(12) (2003) 7874.

[4] A.V. Simakin, et al., "Nanodisks of $\mathrm{Au}$ and Ag produced by laser ablation in liquid environment", Chem. Phys Letters 348 (2001) 182.

[ 5 ] D. Poondi and J singh, "Synthesis of metastable silvernickel alloys by a novel laser-liquid-solid interaction technique", J. Mater. Sci. 35(10) (2000) 2467.

[6] J. Zhang, et al., "Laser-assisted synthesis of superparamagnetic Fe@Au core-shell nanoparticles", J. Phys. Chem. B 110(14) (2006) 7122.

[ 7 ] P. Lui, et al., "Room temperature synthesized rutile $\mathrm{TiO}_{2}$ nanoparticles induced by laser ablation in liquid and their photocatalytic activity", Nanotechnology 20 (2009) 285707.

[ 8 ] K. Amikura, et al., "Copper oxide particles produced by laser ablation in water", Applied Surface Science 254 (2008) 6976

[9] J. Chen, et al., "The irradiation effect of a Nd-YAG pulsed laser on the $\mathrm{CeO}_{2}$ target in the liquid", Materials Letters 58 (2004) 337.

[10] C.C. Huang and C.S. Yeh, "Laser ablation synthesis of spindle-like gallium oxide hydroxide nanoparticles with the presence of cationic cetyltrimethylammonium bromide", J. Phys. Chem. B 108 (2004) 4940.

[11] T. Seto, et al., "Laser ablation synthesis of monodispersed magnetic alloy nanoparticles", J. Nano. Res. 8 (2006) 371.

[12] J. Jakobi, et al., "Magenetic alloy nanoparticles from laser ablation in cyclopentanone and their embedding into a photoresist", Langmuir 26(10) (2010) 6892.

[13] J. Lin, et al., "Synthesis and characterization of FeCo nanoparticle colloid by pulsed laser ablation in distilled water", $33^{\text {rd }}$ EPS Conf. on Plasma Phys. ECA 31I (2006) 2.087.

[14] B.K. Pandey, et al., "Magnetic colloids by pulsed laser ablation", AIP Conf. Proc. 1374 (2011) 35.

[15] V. Amendola, et al., "Magnetic nanoparticles of iron carbide, iron oxide, iron@iron oxide, and metal iron synthesized by laser ablation in organic solvents", J. Phys. Chem. C. 115 (2011) 5140.

[16] S. Sun, et al., "Monodisperse FePt nanoparticles and ferromagnetic FePt nanocrystal superlattices", Science 287 (2000) 1989.

[17] T.H. Kim, et al., "Nanoparticle assemblies as memristors", Nano Lett. 9 (2009) 2229.

[18] D.S. Kang, et al., "Phase transformation and magnetic properties of $\mathrm{NiFe}$ thin films on $\mathrm{Si}(100)$ wafer and $\mathrm{SiO}_{2} /$ $\mathrm{Si}(100)$ substrate by co-sputtering", Journal of the Korean Crystal Growth and Crystal Technology 20(5) (2010) 216.

[19] K.H. Choi, et al., "Fabrication of various carbon nanostructures by using different catalysts", Journal of the Korean Crystal Growth and Crystal Technology 20(3) (2010) 133.

[20] U.I. Tromsdorf, et al., "A highly effective, nontoxic $\mathrm{T}_{1}$ MR contrast agent based on ultrasmall PEGylated iron oxide nanoparticles", Nano. Lett. 9(12) (2009) 4434.

[21] S. Laurent, et al., "Magnetic iron oxide nanoparticles: synthesis, stabilization, vectorization, physicochemical characterizations, and biological applications", Chem. Rev. 108(6) (2008) 2064.

[22] S. Laurent, et al., "Magnetic fluid hyperthermia: Focus on superparamagnetic iron oxide nanoparticles", Advances in Colloid and Interface Science 166 (2011) 8.

[23] M. Chirita and I. Grozescu, " $\mathrm{Fe}_{2} \mathrm{O}_{3}$ nanoparticles, physical properties and their photochemical and photoelectrochemical applications", Cem. Bull. "POLITEHNICA" Univ. (Timisoara) 54(68) (2009) 1.

[24] A. Baladi and R.S. Mamoory, "Study on wavelength and energy effects on pulsed laser ablation synthesis of aluminum nanoparticles in ethanol", Fifth International Conference on MEMS NANO, and Smart Systems (ICMENS) (2009) 218.

[25] X.P. Zhu, et al., "Underwater laser ablation approach to fabricating monodisperse metallic", Chem. Phys Letters 427 (2006) 127.

[26] J.H. Yoo, et al., "Explosive change in crater properties during high power nanosecond laser ablation of silicon", J. Appl. Phys. 88(3) (2000) 1638.

[27] L. Torrisi, et al., "Energy distribution of particles ejected by laser-generated aluminium plasma", Nucl. Instr. and Meth. In Phys. Res. B 252 (2006) 183.

[28] J.M. Fishburn, et al., "Study of the fluence dependent interplay between laser induced material removal mechanisms in metals: Vaporization, melt displacement and melt ejection", Applied Surface Science 252 (2006) 5128.

[29] A.B. Gojani, et al., "Extend measurement of crater depths for aluminum and copper at high irradiances by nanosecond visible laser pulse", Applied Surface Science 252 (2008) 2777.

[30] C. Presnall, Mineral Physics and Crystallography (1995) 248.

[31] D.M. Sherman, "Electronic spectra of Fe3+ oxides and oxide hydroxides in the near IR to near UV", American Mineralogist. 70 (1985) 1262.

[32] G. Kataby, et al., "Characterization of self-assembled alcohols coatings on amorphous iron", Nanostructured Materials 12 (1999) 421. 